\title{
Modified optical OR and AND gates
}

\author{
Avireni Srinivasulu \\ Department of Communication Technology, Defence University Engineering College, \\ Post Box No. 1771, 1110 Addis Ababa, Ethiopia \\ E-mail:avireni_s@yahoo.com
}

\begin{abstract}
This paper deals with optical OR and AND gate, using unijunction transistor (UJT), light emitting diode (LED), and photo-resistor (LDR). Effort is made to extend the development of the gates using UJT, LDR, and LED to work at $1.8 V_{d c}$ instead of $3 V_{d c}$. The power dissipation is approximately $2 \mathrm{~mW}$. These optical gates find application in the field of instrumentation, optical logic isolators, and fiber optics systems where intrinsic safety is of prime importance rather than speed of operation.
\end{abstract}

Keywords: bistability, intrinsic safety, low speed.

Paper received 24.10.02; accepted for publication 17.12.02.

\section{Introduction}

In the last few years attempts ${ }^{1-4}$ have been made towards study and development of fundamental optical gates. Recently, great interest is being taken in developing various optical systems ${ }^{6}$ that provide bistability, because of their practical application in the field of fiber optics and other instrumentation system.

The most recent development ${ }^{5}$ is OR, AND, NOT, NAND, and NOR gates using photoresistor and light emitting diode working at $3 V_{d c}$. In this paper, effort is made to extend the development of these gates using UJT, LED, and LDR to work at $1.8 V_{d c}$. These gates are intrinsically safe and can be used for monitoring of data in hazardous environments such as petroleum industry, chemical industry, fertilizer industry, and underground coalmines ${ }^{6}$.

The UJT used is 2 N2646. The LDR dark resistance is $150 \mathrm{M} \Omega$, and no-dark resistance is $10 \mathrm{~K} \Omega$, Logic 1 and Logic 0 are represented by LED on and LED off.

\section{Optical NOT}

Fig. 1 shows the circuit diagram of optical NOT with input having optical power $O P_{i}$. The photoresistor LDR is connected in series to UJT emitter and the power supply $+V_{d c}$ to the base $B_{2}$ through a pair of resistances $R_{21}$ and $R_{22}$. The pair is selected as to provide required voltage/current to the UJT and LED. The approximate values of $R_{21}, R_{22}, R_{11}$, and $R$ are taken as $100 \Omega, 510 \Omega$, $4.6 \mathrm{~K} \Omega$, and $10 \Omega$ with $+V_{d c}$ equal to $1.8 V_{d c}$. Optical output $O P_{0}$ of NOT occurs only when the input $O P_{i}$ is low, thus satisfying the condition, $O P_{0}=O P_{i}$.

\section{Optical OR}

Fig. 2 shows the circuit diagram of optical OR with input having optical power $O P_{i 1}$ and $O P_{i 2}$. The $\mathrm{LDR}_{1}$ and $\mathrm{LDR}_{2}$ are connected in parallel to the $\mathrm{UJT}_{1}$ emitter and the power supply $+V_{d c}$ to the base $B_{2}$ through a pair of resistances $R_{21}$ and $R_{22}$. The pair is selected to provide required voltage /current to the UJT's and LED's. The approximate values of $R_{21}, R_{22}, R_{11}$, and $R$ are taken as $100 \Omega, 510 \Omega, 4.6 \mathrm{~K} \Omega$, and, $10 \Omega$ with $+V_{d c}$ equal to $+1.8 V_{d c}$. Optical output $O P_{0}$ of OR will not occur when both the inputs $O P_{i 1}$ and $O P_{i 2}$ are low. Thus, satisfied is the condition $O P_{0}=\left(O P_{i 1}+O P_{i 2}\right)$.

\section{Optical AND}

Fig. 3 shows the circuit diagram of optical AND with input having optical power $O P_{i 1}$ and $O P_{i 2}$. The $\mathrm{LDR}_{1}$ and $\mathrm{LDR}_{2}$ are connected in series to the $\mathrm{UJT}_{1}$ emitter and the power supply $+V_{d c}$ to the base $B_{2}$ through a pair 


\section{Avireni Srinivasulu: Modified optical OR and AND gates}
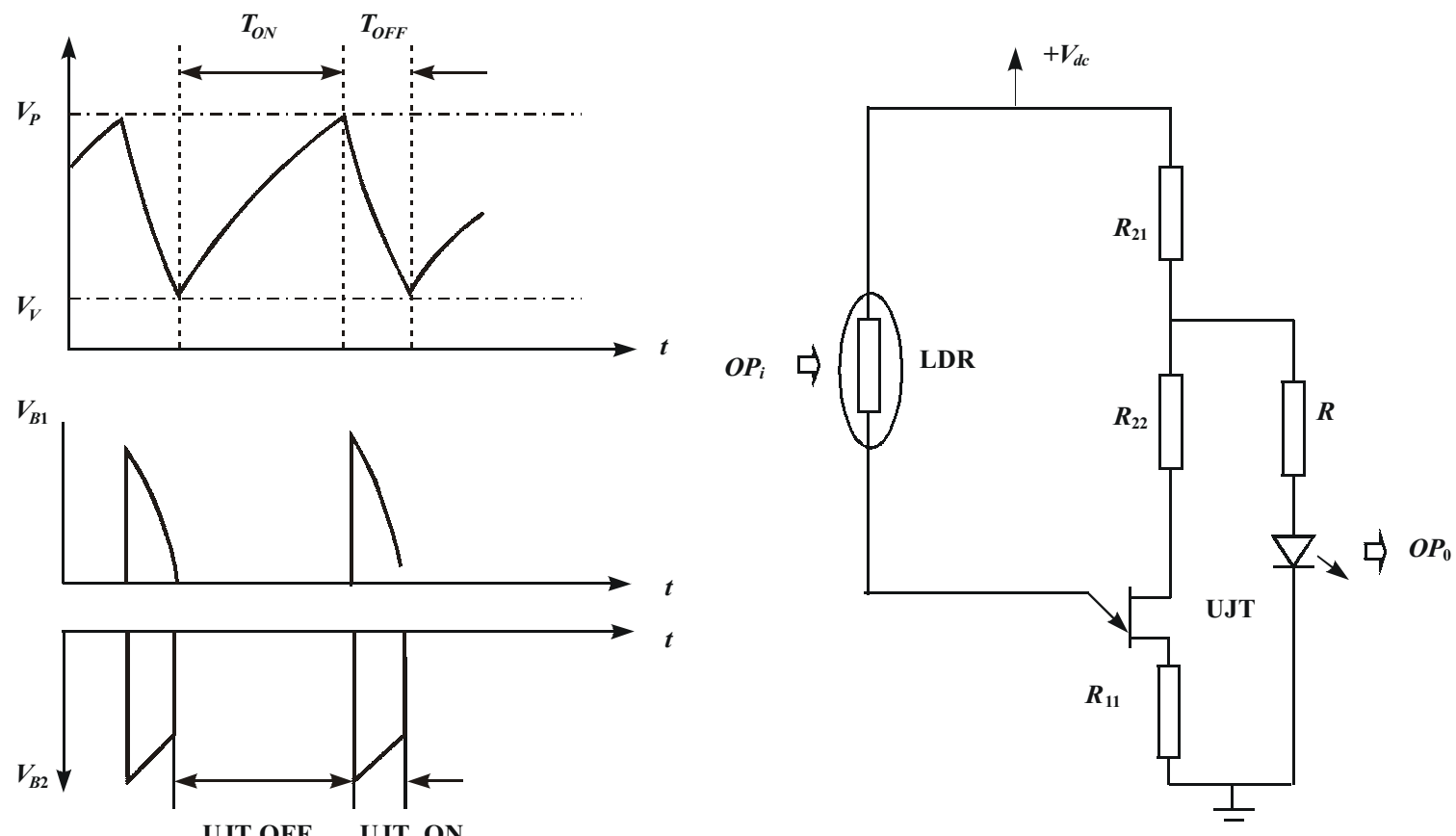

Fig. 1. Optical NOT

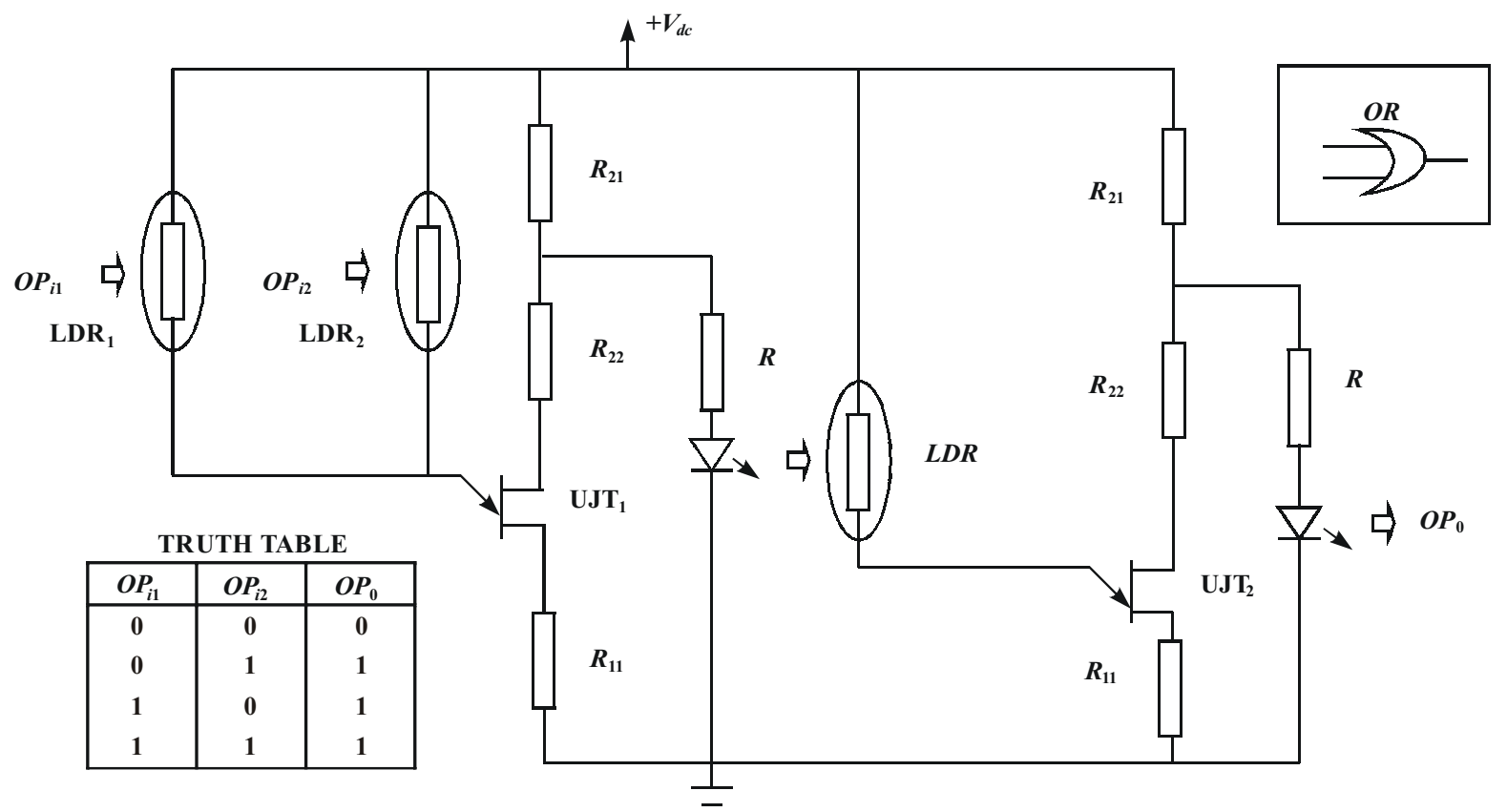

Fig. 2. Optical OR

of resistances $R_{21}$ and $R_{22}$. The pair is selected to provide required voltage /current to the UJT's and LED's. The approximate values of $R_{21}, R_{22}, R_{11}$ and $R$ are taken as $100 \Omega, 510 \Omega, 4.6 \mathrm{~K} \Omega$, and $10 \Omega$ with $+V_{d c}$ equal to +1.8 $V_{d c}$. Optical output $O P_{0}$ of AND will occur only when both the inputs $O P_{i 1}$ and $O P_{i 2}$ are high, thus satisfying the condition $O P_{0}=\left(O P_{i 1} \cdot O P_{i 2}\right)$.
The power dissipation is approximately $2 \mathrm{~mW}$, which is well below intrinsic safe region of highly flammable gases. Fig. 4 shows the experimental curve for LDR resistance against the output voltage.

These circuits though not fast, but finds useful in hazardous environment, where speed is not important. Some of the applications are monitoring system ${ }^{7}$ of $\mathrm{CH}_{4}$ and $\mathrm{CO}_{2}$ in underground coalmine, ethylene and acetylene 


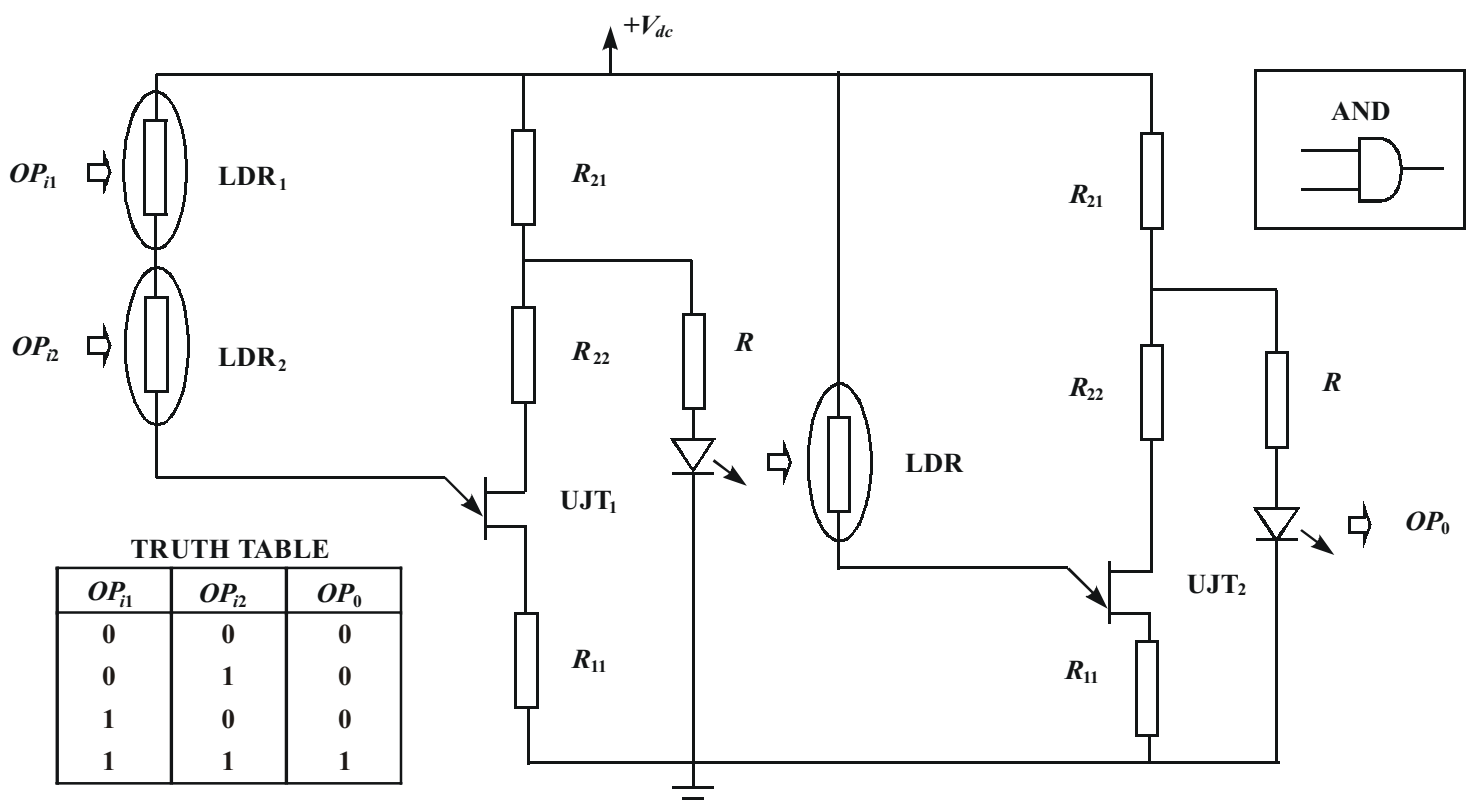

Fig. 3. Optical AND

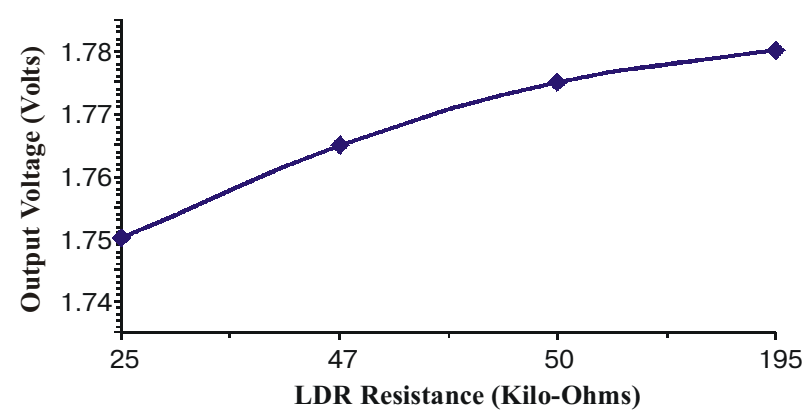

Fig. 4. Output voltage vs LDR resistance

monitoring system in petrochemical industry, monitoring of different hydrocarbons, and gases like $\mathrm{NO}_{\mathrm{X}}, \mathrm{SO}_{2}$ and $\mathrm{CS}_{2}$ in chemical, pharmaceutical cosmetic, and fertilizer industry ${ }^{1,6}$.

\section{Conclusions}

Optical OR and AND were realized successfully using UJT, LED, and LDR working at 1.8 Volts. Fiber optic systems and other areas mentioned above can employ these optical gates for their intrinsic safety, because of low power dissipation. These are incapable as elements in integrated structure. Other gates, multiplexers, and demultiplexers can be established similarly.

\section{References}

1. K.Okumura, Y.Ogawa, H.Ito and H Inaba, IEEE Journal Quantum Electron, vol 21, 1985, p 377.

2. H.Kawaguchi, International Journal of Optoelectron, vol 7, 1992, p302.

3. B.S.Wherett, Y.T.Chow, K.Rhoomy-darzi and A.D.Lloyd, Phys Scr, vol T25, 1989, p247.

4. P.Wheatley. Opt Quantum Electron Lett, vol 19, 1987, p 267.

5. V.Kumar and P.K.Roy, Journal of Opt Applications, vol 25, no 2, 1995.

6. P.K.Roy and Dr.N.Roy, Journal of the institution of engineers (India), vol 82, 2002, p 55.

7. V.Kumar and G.M.Prasad, 'Mining Technol', vol 71, 1987, p 187.

8. K.Otsuka, Electron Lett, vol 24, 1988, p 800. 